\title{
Control modeling of the fast-steering secondary mirror for GMT
}

Christoph Dribusch, Myung K. Cho, Youra Jun, Jieun Ryu, Gary Poczulp, et al.

Christoph Dribusch, Myung K. Cho, Youra Jun, Jieun Ryu, Gary Poczulp, Ming Liang, Sungho Lee, Jeong-Yeol Han, Ueejeong Jeong, Sanghyuk Kim, Bong-Kon Moon, Chang-Hee Kim, Yunjong Kim, Chan Park, ByeongGon Park, Il-Kwon Moon, Chanhee Lee, Wongi Lee, Ho-Sang Kim, Paul Gardner, Robert Bernier, Frank Groark, Hugo Chiquito, "Control modeling of the fast-steering secondary mirror for GMT," Proc. SPIE 10705, Modeling, Systems Engineering, and Project Management for Astronomy VIII, $107050 \mathrm{Z}$ (10 July 2018); doi: 10.1117/12.2313351

EDent: SPIE Astronomical Telescopes + Instrumentation, 2018, Austin, Texas, United States 


\title{
Control Modeling of the Fast-Steering Secondary Mirror of GMT
}

\author{
Christoph Dribusch*a , Myung K. Cho ${ }^{\mathrm{b}}$, Youra Jun ${ }^{\mathrm{a}}$, Jieun Ryu ${ }^{\mathrm{a}}$, Gary Poczulp ${ }^{\mathrm{b}}$, Ming Liang ${ }^{\mathrm{b}}$, \\ Sungho Lee ${ }^{c}$, Jeong-Yeol Han ${ }^{c}$, Ueejeong Jeong ${ }^{c}$, Sanghyuk Kim ${ }^{c}$, Bong-Kon Moon ${ }^{c}$, \\ Chang-Hee Kim ${ }^{\mathrm{c}}$, Y unjong Kim ${ }^{\mathrm{c}}$, Chan Park ${ }^{\mathrm{c}}$, Byeong-Gon Park ${ }^{\mathrm{c}}$, \\ Il-Kwon Moon ${ }^{\mathrm{d}}$, Chanhee Lee $\mathrm{e}^{\mathrm{e}}$, Wongi Lee ${ }^{\mathrm{e}}$, Ho-Sang Kim ${ }^{\mathrm{e}}$, \\ Paul Gardner ${ }^{\mathrm{f}}$, Robert Bernier ${ }^{\mathrm{f}}$, Frank Groark ${ }^{\mathrm{f}}$, Hugo Chiquito ${ }^{\mathrm{f}}$ \\ ${ }^{a}$ University of Arizona, Tucson, AZ 85721, USA; \\ ${ }^{b}$ National Optical Astronomy Observatory, 950 N. Cherry Ave., Tucson, AZ 85719, USA; ${ }^{c}$ Korea \\ Astronomy and Space Science Institute, Daejeon 34055, Republic of Korea; \\ ${ }^{\mathrm{d} K o r e a ~ R e s e a r c h ~ I n s t i t u t e ~ o f ~ S t a n d a r d s ~ a n d ~ S c i e n c e, ~ D a e j e o n ~ 34113, ~ R e p u b l i c ~ o f ~ K o r e a ; ~}$ \\ 'Institute for Advanced Engineering, Yongin-si, Gyeonggi-do 17180, Republic of Korea; \\ ${ }^{\mathrm{f}}$ GMTO Corporation, 465 N. Halstead Street, Suite 250, Pasadena, CA 91107, USA
}

\begin{abstract}
The Giant Magellan Telescope (GMT) will feature two Gregorian secondary mirrors, an adaptive secondary mirror (ASM) and a fast-steering secondary mirror (FSM). The FSM has an effective diameter of $3.2 \mathrm{~m}$ and consists of seven $1.1 \mathrm{~m}$ diameter circular segments, which are conjugated $1: 1$ to the seven $8.4 \mathrm{~m}$ segments of the primary. Each FSM segment contains a tip-tilt capability for fast guiding to attenuate telescope wind shake and mount control jitter. This tiptilt capability thus enhances performance of the telescope in the seeing limited observation mode. The tip-tilt motion of the mirror is produced by three piezo actuators. In this paper we present a simulation model of the tip-tilt system which focuses on the piezo-actuators. The model includes hysteresis effects in the piezo elements and the position feedback control loop.
\end{abstract}

Keywords: Piezo Actuator, Giant Magellan Telescope, Fast Steering Secondary Mirror, opto-mechanics, mirror support, tip-tilt, control model

\section{INTRODUCTION}

The Giant Magellan Telescope (GMT) will feature two Gregorian secondary mirrors, an adaptive secondary mirror (ASM) and a fast-steering secondary mirror (FSM). The FSM has an effective diameter of $3.2 \mathrm{~m}$ and consists of seven $1.1 \mathrm{~m}$ diameter circular segments, which are conjugated $1: 1$ to the seven $8.4 \mathrm{~m}$ segments of the primary. Each FSM segment contains a tip-tilt capability for fast guiding to attenuate telescope wind shake and mount control jitter as shown in Figure 1. This tip-tilt capability thus enhances performance of the telescope in the seeing limited observation mode. The tip-tilt motion of the mirror is produced by three piezo actuators. The tip-tilt system needs to follow a reference signal for tip and tilt angles, which is based on wave-front measurements. It is also essential that the reaction forces applied by the mirror to the top-end of the telescope do not cause vibrations that would eliminate the benefit of the tiptilt system. There is no active compensation for these reaction forces. This paper presents the current iteration of a simulation model of the tip-tilt system that aims to predict its tracking performance as well as the reaction forces onto the telescope top-end. Figure 2 shows the piezo actuator of FSM2 in detail. It features a piezo element that is pre-loaded by external springs to enable push and pull. The preload springs ensure that the piezo element is always in compression. This is essential to prevent damage. The model of the piezo element relies heavily on work by Jiri Figura ${ }^{2}$.

Modeling, Systems Engineering, and Project Management for Astronomy VIII edited by George Z. Angeli, Philippe Dierickx, Proc. of SPIE Vol. 10705

$107050 Z$ - (c) 2018 SPIE · CCC code: 0277-786X/18/\$18 

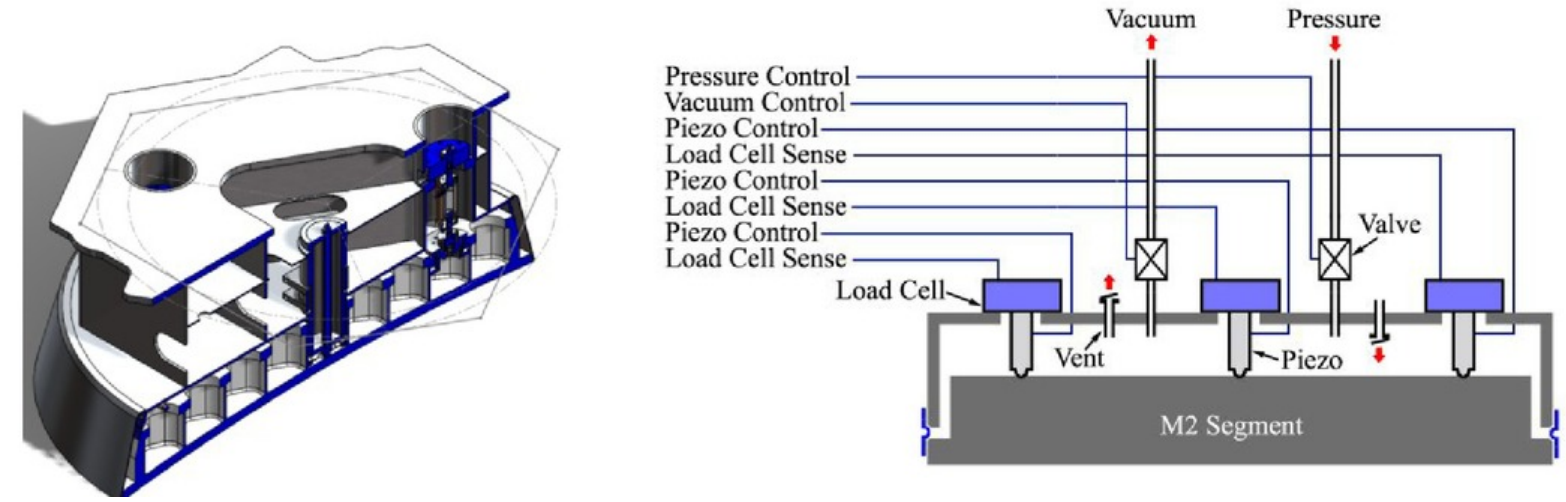

Figure 1. Segment of the GMT FSM2. The mirror cell and mirror are shown on the left and the right shows a diagram of the piezo actuators and the vacuum gravity compensation.

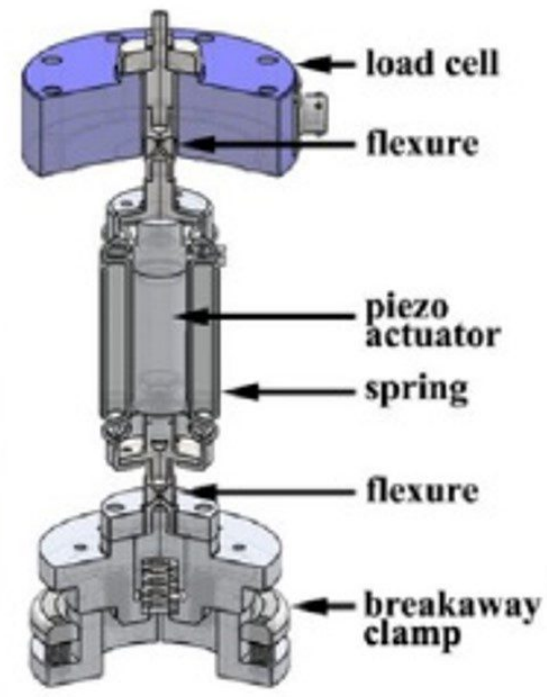

Figure 2. Piezo actuator of FSM. The piezo element is PI-845.30 manufactured by Physik Instrumente. External preload springs ensure that the piezo element is always in compression, even when the actuator pulls on the mirror.

\subsection{Model}

The simulations are performed in Simulink and Figure 3 shows an overview of the model. The mirror has two rotation axes, where the $\mathrm{Y}$-axis is a symmetry axis and the $\mathrm{X}$-Axis is not. Reference values for tip and tilt and piston are provided as input. The piston reference signal is always zero. A linear transform converts the mirror reference signals to equivalent actuator displacements. The actuator displacement error signal is fed to three separate output-limited PI controllers that generate the driving voltage for the three piezo elements. This is a simplification, because realistically the output of the controller would have to be offset and amplified. However, the Simulink model of the piezo actuator element is compatible with this simplification. The piezo elements displace the mirror which in turns exerts reaction forces. Position feedback from the strain gages of the piezo elements is used to generate the position error signal fed into the PI controllers. The mirror uses a linear transform to convert accelerations to reaction forces based on mass and moment of inertia of the mirror. Likewise another linear transform converts actuator displacements to piston, tip and tilt values. The model of the piezo elements is more sophisticated and described in detail in the following section. 


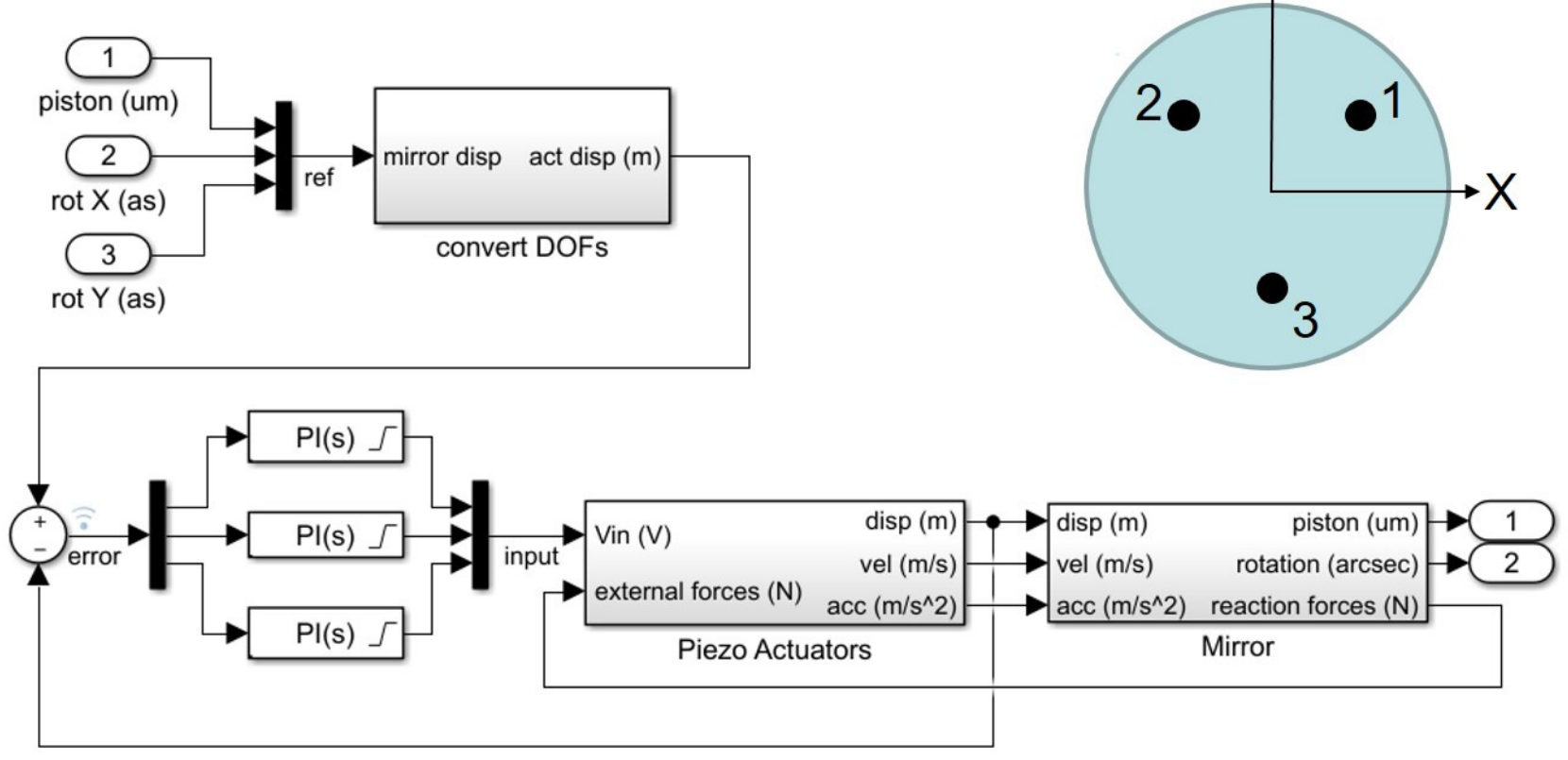

Figure 3. Overview of the tip-tilt control system. Also shown are the two rotation axes: The Y-axis is a symmetry axis, while the $\mathrm{X}$-axis is not.

\section{MODEL OF PIEZO ELEMENT}

The piezo actuators block contains 3 identical piezo actuators. As shown in Figure 4, each actuator is modeled as a simple $2^{\text {nd }}$ order equation of motion with mass, damping and stiffness. In addition a nonlinear hysteresis feedback loop ensures that the piezo element expresses realistic hysteresis. In an open-loop configuration this hysteresis of the piezo element would lead to large error in the rotation of the mirror. The input of the hysteresis block is the electric charge of piezo element and the output is the hysteresis voltage that is subtracted from the input voltage driving the piezo element. 


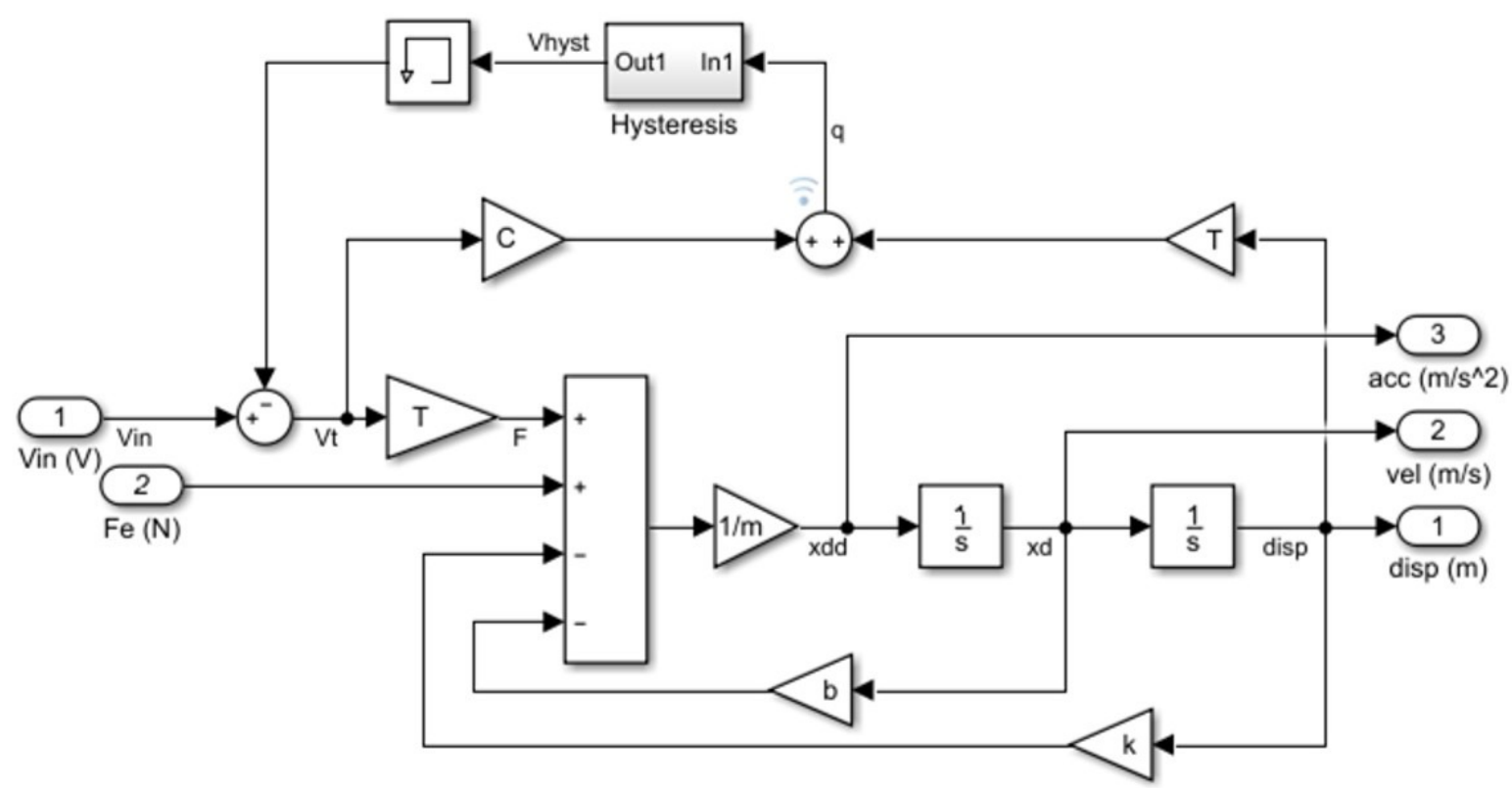

Figure 4. Overview of the tip-tilt control system. Also shown are the two rotation axes: The Y-axis is a symmetry axis, while the $\mathrm{X}$-axis is not.

The hysteresis block uses a Maxwell model ${ }^{2}$. The general concept is that a simple combination of backlash and gain as shown in Figure 5 exhibits hysteresis. Putting a number of such combinations in parallel and taking the sum, allows for a piece-wise linear approximation of a given hysteresis curve (Figure 6). A simple formula exists select parameters $\mathrm{k}_{\mathrm{i}}$ and $\mathrm{w}_{\mathrm{i}}$ to approximate a given hysteresis curve ${ }^{2}$.
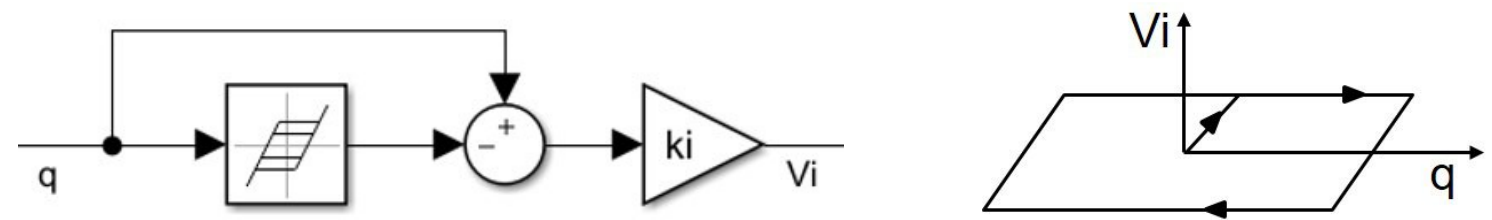

Figure 5. Simple combination of backlash and gain blocks forms a single Maxwell hysteresis element.
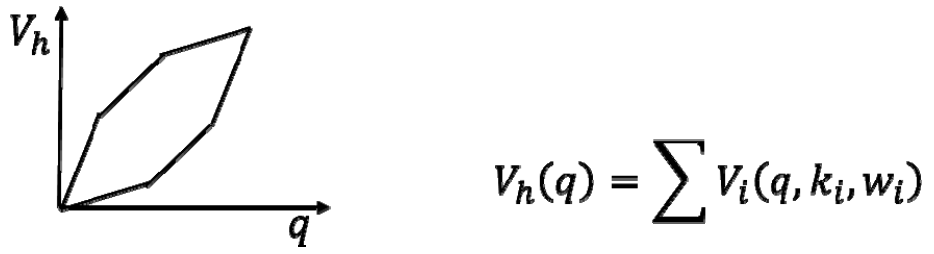

Figure 6. A sum of parallel Maxwell hysteresis elements will approximate a given hysteresis curve in a piece-wise linear fashion.

For this paper 15 Maxwell hysteresis blocks per actuator were combined to obtain the hysteresis curves shown in Figure 7. 

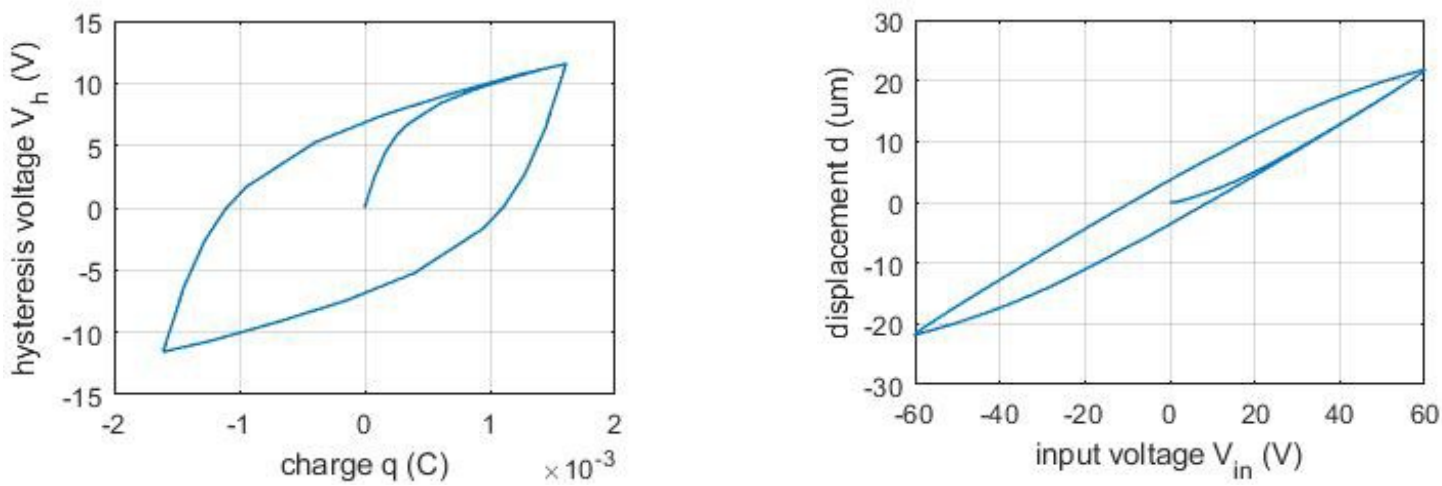

Figure 7. A combination of 15 Maxwell hysteresis elements yields this open-loop actuator displacement under zero external load.

\section{SIMULATION RESULTS}

In order to simulate the system, parameters for the PI controllers need to be selected. To select these parameters the model was linearized and the parameters P and I were chosen to obtain an overshoot of $20 \%$ and a phase margin of 38 degrees. The same parameters were used for every PI controller.

The reference trajectory for piston, tip and tilt was selected as zero for piston and rotation about the Y-axis (Figure 3) and a sine wave for rotation about the $\mathrm{X}$-axis (Figure 8). The amplitude of the sine wave is ramped up to prevent a discontinuity in acceleration at time $=0$.
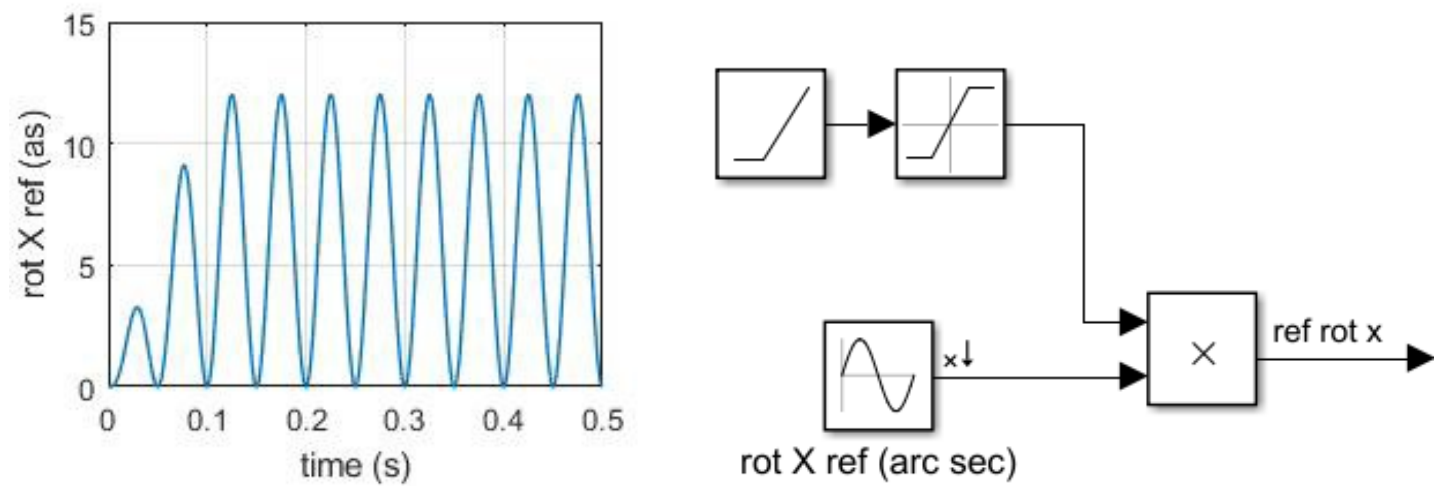

Figure 8. Reference signal for $\mathrm{x}$-axis rotation as used in the simulations.

Figures 9 to 11 show the simulation results without taking Piezo hysteresis into account, while Figures 12 to 14 show the simulation results with hysteresis taken into account. Without hysteresis the error in the mirror degrees of freedom with zero reference signal is also zero, but with hysteresis that is no longer the case. Another notable difference is that the results with hysteresis show higher frequencies in the reaction force response. However the maximum position errors and peak reaction forces are comparable. 


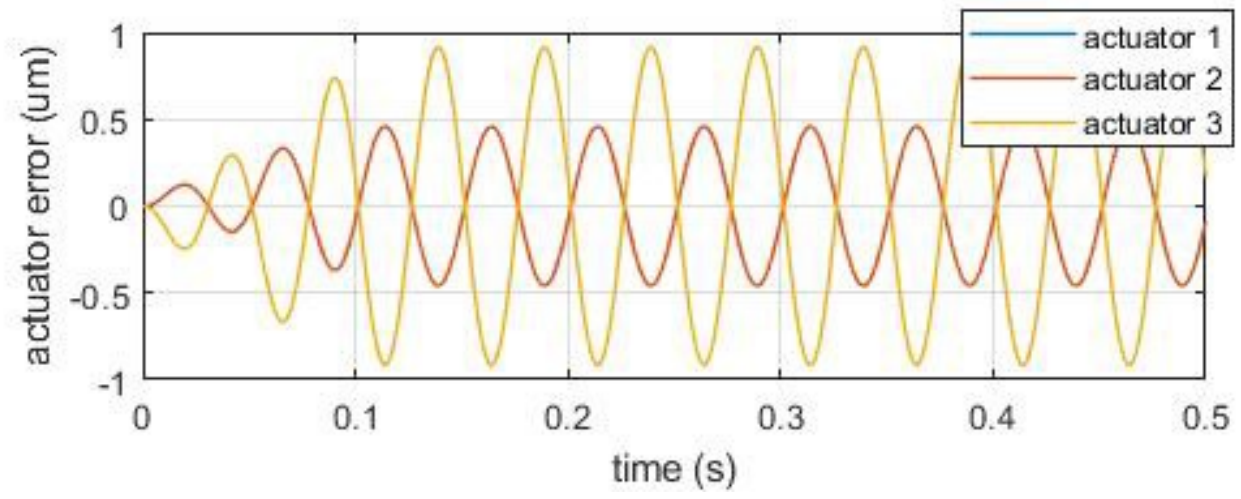

Figure 9. Without piezo hysteresis: Displacement error of actuators. Errors of actuator 1 and 2 are identical.

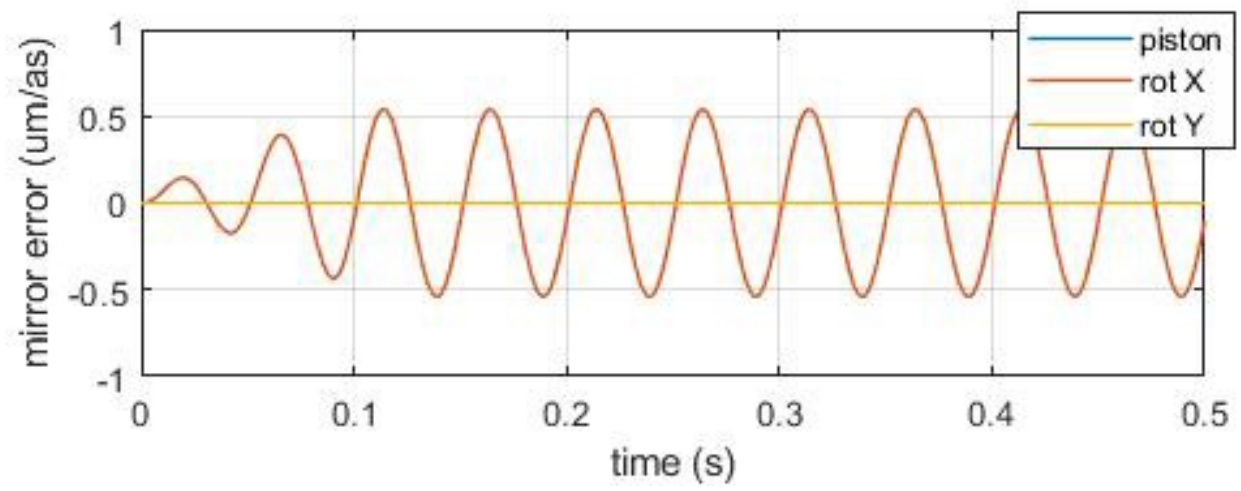

Figure 10. Without piezo hysteresis: Mirror displacement error. The piston and rot $\mathrm{Y}$ errors are zero.

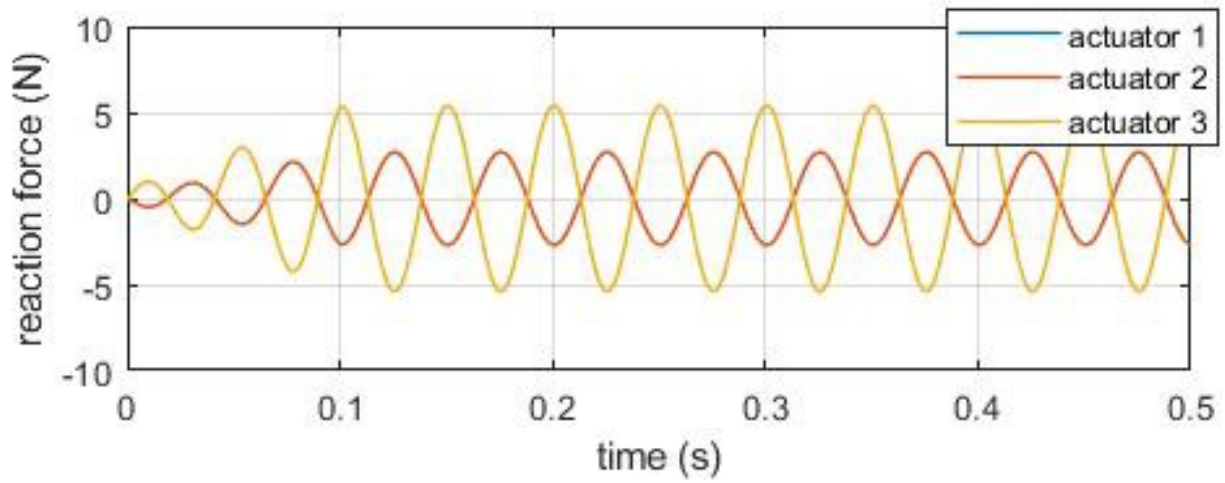

Figure 11. Without piezo hysteresis: Actuator reaction forces exerted onto the mirror cell. Actuators 1 and 2 are identical. 


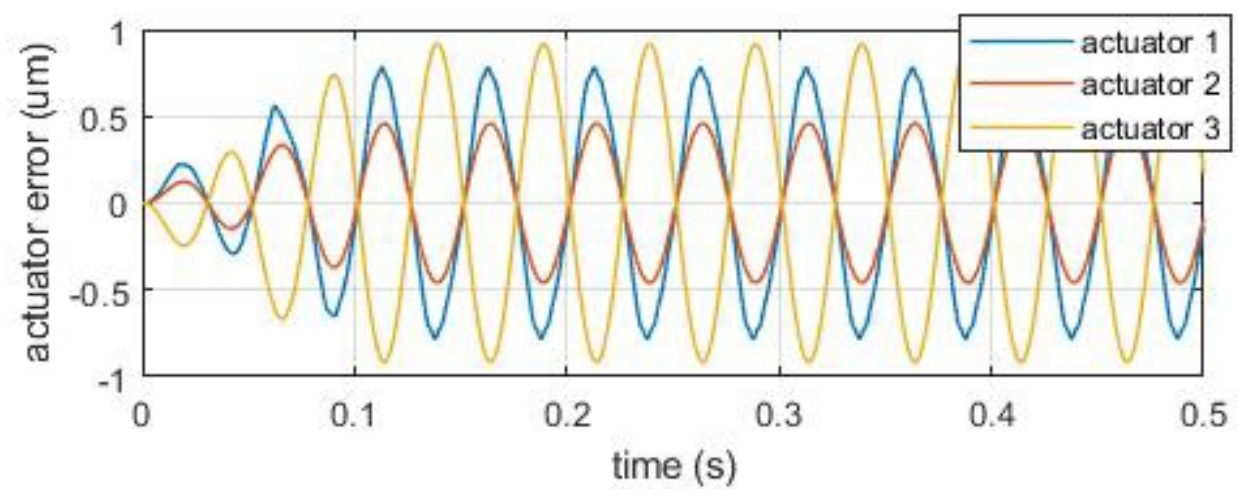

Figure 12. With piezo hysteresis: Displacement error of actuators.

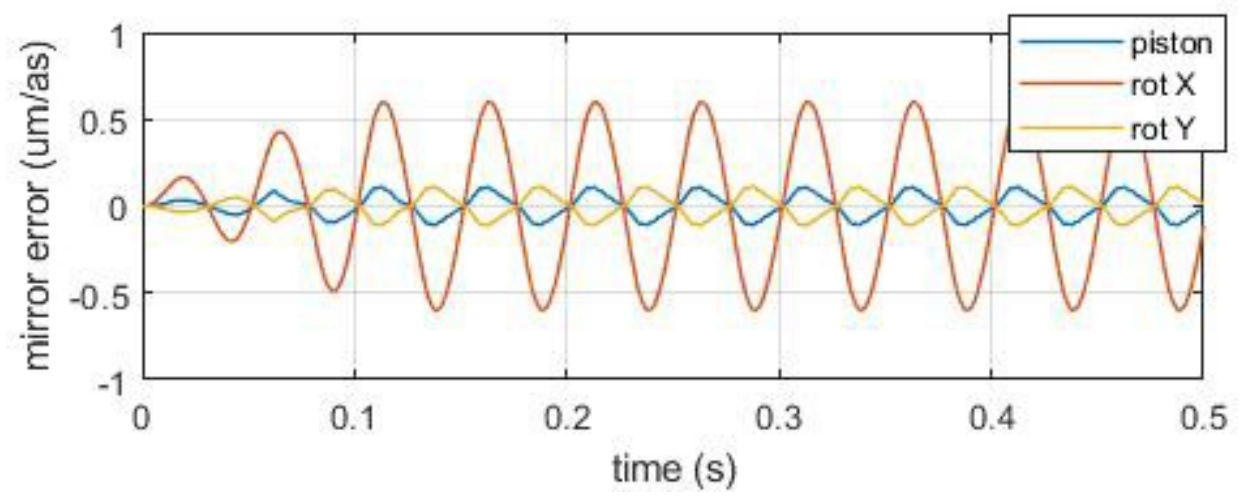

Figure 13. With piezo hysteresis: Mirror displacement error.

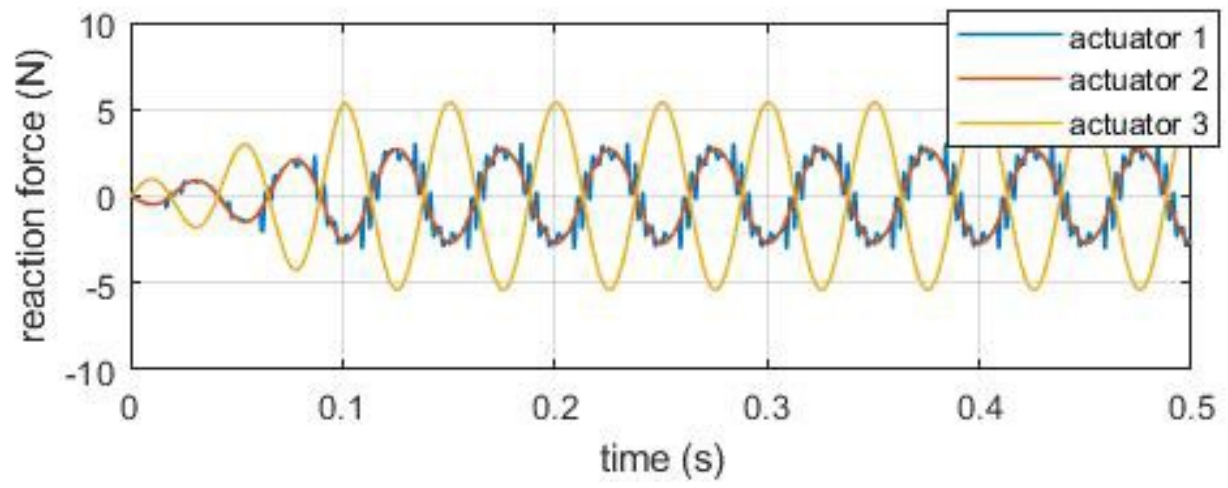

Figure 14. With piezo hysteresis: Actuator reaction forces exerted onto the mirror cell.

\section{CONCLUSIONS}

The Maxwell model of piezo element hysteresis is easy to implement in simulations and its parameters can be obtained from the open-loop response of the actuators. While the effect of the hysteresis is not very large, it is noticeable in the position error of the mirror surface as well as in the frequency content of the reaction forces. It is also noteworthy that some of the other piezo parameters such as damping and element mass only had a very small effect on the results. 


\section{REFERENCES}

[1] Figura, J., [Bachelor Project: Modeling and Control of Piezoelectric Microactuators], Czech Technical University in Prague, 26-28 (2013)

Proc. of SPIE Vol. 10705 107050Z-8

Downloaded From: https://www.spiedigitallibrary.org/conference-proceedings-of-spie on 12/13/2018 Terms of Use: https://www.spiedigitallibrary.org/terms-of-use 